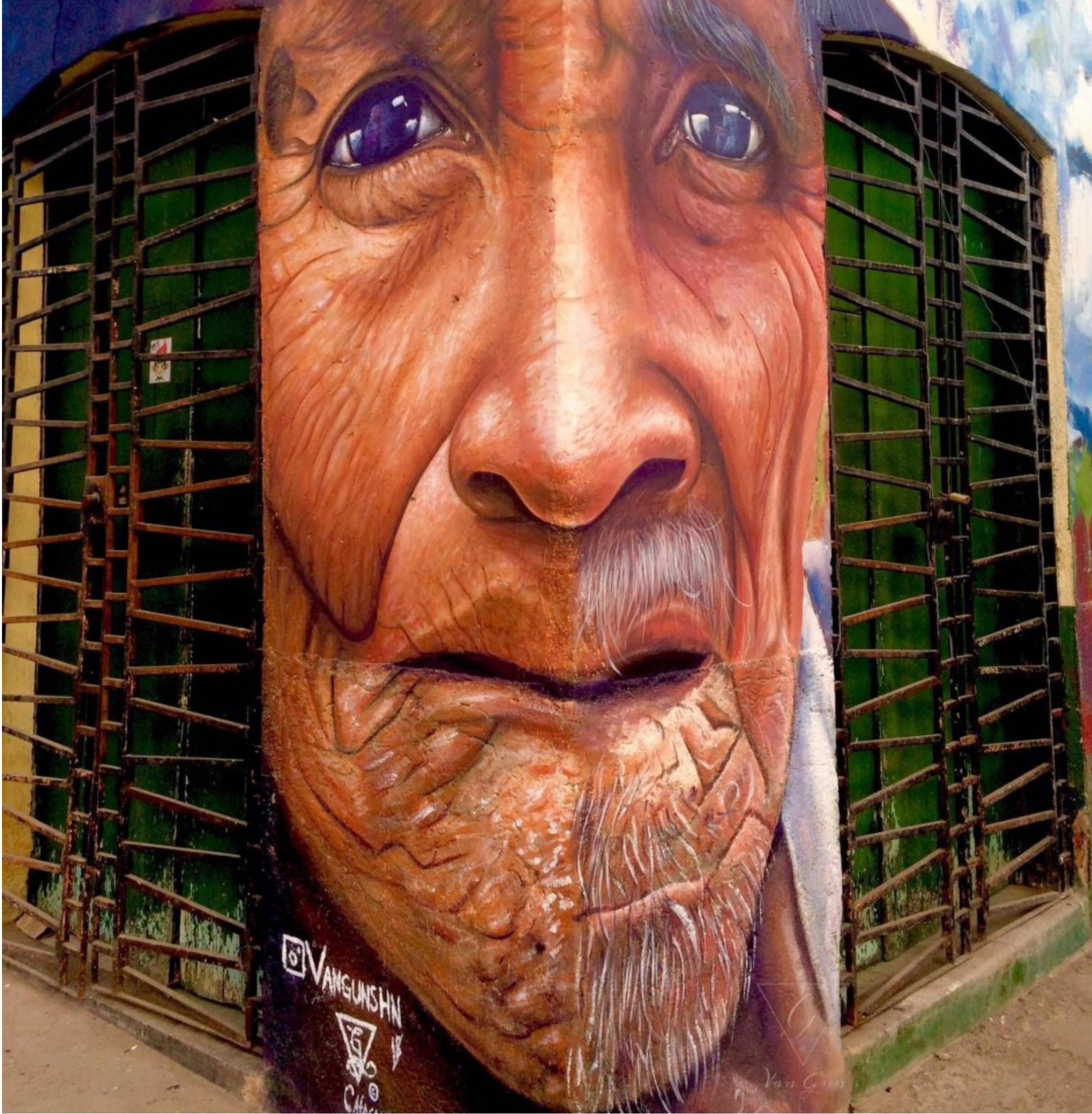




\section{LA MIGRANTE}

Mujer que caminas noche y día con tu llave inmemorial das nacimiento a la palabra veraz atraviesas el río y nadie te reconoce

te mojas, sudas, pierdes tus zapatos.

Otra jornada abrumada de cansancio no puedes verbalizar tus injurias pero eres sabia, aunque te encuentren infraganti.

Te interrogan

¿Quién eres? ¿De dónde vienes?

¿Qué buscas en esta tierra que ya tiene dueños

$y$ fronteras y murallas y hermanos que saben de la muerte lenta?

Al espacio de tu linaje vuelves como sombra que releo en tu luminosa faz el fuego no termina escapas, caes, te levantas, te sacudes, hablas en tu lengua de tortilla muerdes tus palabras de café y no te dejas derrotar por la nostalgia...

Tu canto se ahoga, se alejan las salidas eres inmigrante tu identidad se ha reducido para siempre.

No entiendes de visas ni de planetas fragmentados aprendes a decir "good morning" pero a nadie le interesa "how you are" ni que estés habitada por un hijo sepultado en el desierto por el sueño de un empleo de un refugio para dormir en paz.

Yo también soy la ruptura de la costura aquí adentro no se disipa la niebla... y me sucede que miro en tu espejo y me veo. 


\title{
1. Migraciones internacionales, familias y género
}

\author{
María Eugenia Zavala de Cosío ${ }^{1}$ \\ DOI: https://doi.org/10.5377/pdac.v88i2.12734 \\ Recibido: 16/04/2021 - Aceptado: 20/05/2021
}

\begin{abstract}
Resumen: Las investigaciones sobre las migraciones internacionales se interesan cada vez más a las transformaciones familiares, dada la mayor visibilidad de los flujos femeninos en el mundo y los problemas específicos de las mujeres migrantes. Este artículo se centra en la influencia de las relaciones del género sobre las dinámicas sociodemográficas, con el objetivo de analizar de manera conjunta las trayectorias migratorias y familiares. La metodología es doble: primero, se investiga si hay una feminización de las migraciones en América Latina, basándose en las estimaciones de la División de Población de las Naciones Unidas, entre 2003 y 2019. Y posteriormente, para analizar las trayectorias de vida familiares y de género en el contexto de las migraciones, se revisan varios estudios empíricos de casos latinoamericanos que tienen esa perspectiva. Como resultados de los análisis, se llega a la conclusión de que no hay una feminización general de las migraciones en América Latina, pero que para explicar las características de las migraciones de mujeres es preciso identificar las variables determinantes de las relaciones entre las migraciones, las familias y el género. Finalmente se propone una herramienta de recolección y análisis de los datos sobre las historias de vida, la ficha AGEVEN, que relaciona las migraciones, la familia y el género. Entre los resultados importantes de este artículo se muestra que, a pesar de las nuevas atribuciones adquiridas durante el proceso migratorio, la desigualdad de género de origen sigue ejerciéndose a través de las fronteras.
\end{abstract}

Palabras claves: migraciones, familia, género, mercado matrimonial, trabajo.

\section{International migration, families and gender}

\begin{abstract}
Research on international migration is interested more and more in family transformations, due to the greater visibility of female flow worldwide and the specific problems faced by migrant women. This article focuses on the influence of gender relations on sociodemographic dynamics, with the aim of analyzing jointly migration and family trajectories. The methodology is twofold: first, it is investigated whether there is a feminization of migrations in Latin America, based on estimates from the United Nations Population Division, between 2003 and 2019. And then, to analyze family and gender life courses in the context of migrations, several Latin American researches are reviewed, with empirical data on this perspective. The conclusions are that even if there is no general feminization of migrations in Latin America, to explain the characteristics of women's migrations, it is necessary to identify the determinant variables explaining the relationships between migration, families and gender. Finally, the AGEVEN tab is proposed, as a tool for collecting and analyzing data on the life courses that relate migrations, family and gender. Among the important results of this article is that, despite new roles obtained during the migration process, gender inequality in societies of origin continues to be exercised across borders.
\end{abstract}

Key words: migration, family, gender, marriage market, labor.

\section{Introducción}

Las investigaciones sobre las migraciones internacionales se interesan cada vez más en las relaciones de género y en las transformaciones familiares, dada la mayor visibilidad de los flujos femeninos en el mundo y de los problemas específicos de las mujeres migrantes. Por un lado, para abordar esa temática, se revisan las estimaciones recientes de las migraciones en América Latina según el sexo, por medio de los datos de la División de las Naciones
Unidas entre 2003 y 2019. Por otro lado, se investiga la integración de las relaciones de género y de las dinámicas familiares con las etapas de los procesos migratorios de las mujeres.

Internacionalmente, las mujeres migrantes están cada vez más presentes y una proporción significativa migra individualmente (Lauby \& Stark, 1987). El concepto de feminización de la migración surgió cuando aumentaron las proporciones de mujeres migrantes en comparación con los hombres (ONU,

1 Doctorado en Demografía de la Universidad de Paris Sorbonne. Maestría de Demografía de El Colegio de México Licenciatura en Relaciones Internacionales y Profesora Investigadora de El Colegio de México, e-mail: mzavala@colmex.mx

Revista Población y Desarrollo: Argonatuas y Caminantes, vol.17, 2021 
2011; Pfeiffer et al., 2007). Aunque estudios comparativos han demostrado que la migración femenina no es un fenómeno reciente (Schrover, 2013; Donato, 2015), se ha visibilizado más a partir de la década de 1980: "Las mujeres comenzaron a ser percibidas como actores sociales y económicos incontestables de su migración" (Vause \& Toma, p. 46). Cuando la mayoría de los migrantes eran varones, rara vez se estudiaban los cambios familiares, porque las variables económicas eran esenciales.

En este artículo, que se centra en las dinámicas sociodemográficas de las mujeres latinoamericanas migrantes, se subraya el vínculo entre las etapas migratorias y las trayectorias familiares. Se intenta descomponer la movilidad espacial, observando cómo se produce una migración en relación con un cambio familiar (causa y/o efecto) y cómo este cambio familiar está relacionado con los cambios en las relaciones de género (causa y/o efecto también). El objetivo es identificar las variables determinantes de las migraciones femeninas para explicar sus características y tendencias. Finalmente, se propone el uso de una ficha AGEVEN, como una herramienta de recolección y de análisis sobre las relaciones entre las migraciones, la familia y el género.

\section{Metodología}

Este artículo se divide en dos partes. La primera examina la evolución de la presencia de las mujeres en la migración internacional entre 2003 y 2019, particularmente en América Latina y el Caribe, con datos de las estimaciones de la División de Población de las Naciones Unidas. Se analizan los stocks acumulados de mujeres migrantes en las diferentes regiones del mundo, las evoluciones de las proporciones de mujeres migrantes en América Latina y el Caribe entre 1960 y 2019 (ONU, 2003, 2011, 2013, 2019), así como los flujos de migraciones femeninas latinoamericanas a nivel internacional.

En la segunda parte, se consideran los resultados de varios estudios específicos sobre las migraciones femeninas latinoamericanas, para identificar las variables determinantes de las relaciones entre las migraciones, las familias y el género. El primer ejemplo de esas interacciones se interesa a los mercados matrimoniales, destacando el aumento de los matrimonios mixtos, entre mujeres migrantes y varones nativos, una consecuencia directa de las desigualdades de género en las sociedades receptoras (Domingo et al., 2014; Roca Girona, 2014). Luego se abordan los temas de la sexualidad en la migración
(Marroni, 2009), de las relaciones de género y del empoderamiento en las familias (Pagnotta, 2014), de las dinámicas familiares (Guimarães \& Baeninger, 2014) y de los mercados de trabajo transnacionales (Acosta \& Petién, 2014). El hilo conductor del análisis es la dimensión familiar de la movilidad, así como los cambios en las relaciones de género.

En el contexto de las migraciones internacionales, se modifican la autonomía y el empoderamiento femenino, los roles y las responsabilidades familiares (Rosas, 2014), la maternidad transnacional a distancia (Cavagnoud, 2014), además del rol de las mujeres como proveedoras económicas de sus familias, responsables de redes migratorias y figuras clave en los procesos reproductivos (Pedone, Echeverri \& Gil, 2014).

La construcción del marco conceptual de las variables determinantes de las migraciones con perspectiva de género se apoya en los resultados de investigaciones empíricas realizadas en América Latina, España y Francia. La base de la reflexión parte de los trabajos presentados y discutidos en el Simposio "Las migraciones femeninas en América Latina y las transformaciones en las relaciones de género", coordinado por María Eugenia Zavala y Virginie Rozée, en el Congreso de Americanistas de Viena (Austria), en 2012. Es un material de gran valor para la síntesis que aquí se propone, ya que representa un conjunto de resultados de investigaciones enfocadas a las relaciones entre las migraciones internacionales, las familias y el género, con una perspectiva común (Zavala de Cosío \& Rozée, 2014). Se añaden investigaciones empíricas realizadas en otros contextos de emigración a los Estados Unidos, como el estudio sobre sexualidad de emigrantes mexicanos desde Puebla (Marroni, 2009), y la entrada a la vida matrimonial de emigrantes guatemaltecos (Aragón, 2014).

Este acervo de resultados empíricos permite construir un esquema de análisis de las variables determinantes de las migraciones, que ordenan la complejidad de las relaciones entre las migraciones, las familias y el género. Los métodos biográficos son los más adecuados para una observación a lo largo del curso de vida de las trayectorias migratorias (con cuestionarios biográficos, historias de vida cualitativas o ambos combinados). $Y$ al final se propone en la Figura $\mathrm{N}^{\circ} 1$ una herramienta de recolección y análisis de datos, la ficha AGEVEN, para realizar encuestas con un método sencillo y ampliamente comprobado (Cavagnoud, Baillet \& Cosío Zavala, 2019). 
Idealmente, los avances metodológicos también deberían aplicarse a los varones y a sus familias, con el fin de realizar análisis más completos de la movilidad espacial en todas sus dimensiones, y hay que alentar la recolección de ese tipo de datos.

\section{Discusión de resultados}

En una primera parte, se discuten las tendencias de las migraciones femeninas, con una mirada comparativa. En la segunda parte, se subrayan las relaciones entre las migraciones, las familias y el género, que conducen a plantear un esquema de variables determinantes de las migraciones. Finalmente, se propone la ficha AGEVEN como una herramienta para el levantamiento de datos y sus análisis.

\subsection{Tendencias de las migraciones femeninas}

\subsection{1. ¿Hubo una feminización de las migraciones en América Latina?}

Para responder a esta pregunta, se presentan estimaciones de la feminización de la migración internacional en América Latina y el Caribe, en comparación con distintas regiones geográficas del mundo, según datos de la División de Población de las Naciones Unidas (ONU, 2003, 2011, 2013, 2019). A finales del Siglo XX, se observó un fuerte flujo migratorio de mujeres desde América del Sur hacia España y Portugal, y un retorno importante con la crisis económica mundial de 2007 hacia los países de origen (como Brasil, Colombia, Ecuador, Perú). También aumentó la proporción de mujeres en las migraciones mexicanas hacia los Estados Unidos y ellas están muy presentes en las migraciones fronterizas entre Brasil, Bolivia, Chile, Colombia, Paraguay, Perú y Venezuela (Zavala de Cosío \& Rozée, 2014). Las migraciones se pueden medir por sexo con los stocks de migrantes o bien según los flujos migratorios.

\section{- Los stocks de inmigrantes}

La población residente en un país distinto de su país de origen permite medir los stocks de migrantes internacionales en cada país o región del mundo, es decir el número de extranjeros inmigrantes en los países de destino. La proporción de mujeres a nivel mundial se mantiene relativamente estable durante el período 1990-2019, ligeramente inferior a la mitad: aumenta entre 1990 (48.8\%) y 2000 (49.3\%), para volver a caer en 2010 y 2019 (48.1\% y $47.9 \%$ respectivamente) (ONU, 2003, 2011, 2013, 2019).
El Gráfico №1 muestra el número y la proporción de mujeres entre los stocks de migrantes en 2019, en las diferentes regiones del mundo. Europa y América del Norte fueron las dos únicas regiones donde las mujeres inmigrantes superan a los varones (ONU, 2019). En 2019, la proporción de mujeres migrantes alcanza $49.9 \%$ en América Latina. Es superior a la mitad en América del Norte (51.8\%) y en Europa (51.4\%); es de $47.0 \%$ en África, $41.5 \%$ en Asia, $50 \%$ en Oceanía. En 2019, del conjunto de migrantes internacionales (272 millones), América Latina y el Caribe incluyen a casi 12 millones de ambos sexos, con 5.8 millones de mujeres (ONU, 2019).

Gráfico $\mathbf{N}^{\circ} \mathbf{1}$ Stock de mujeres migrantes y proporción de mujeres entre el total de migrantes por región geogáfica del mundo. Año 2019

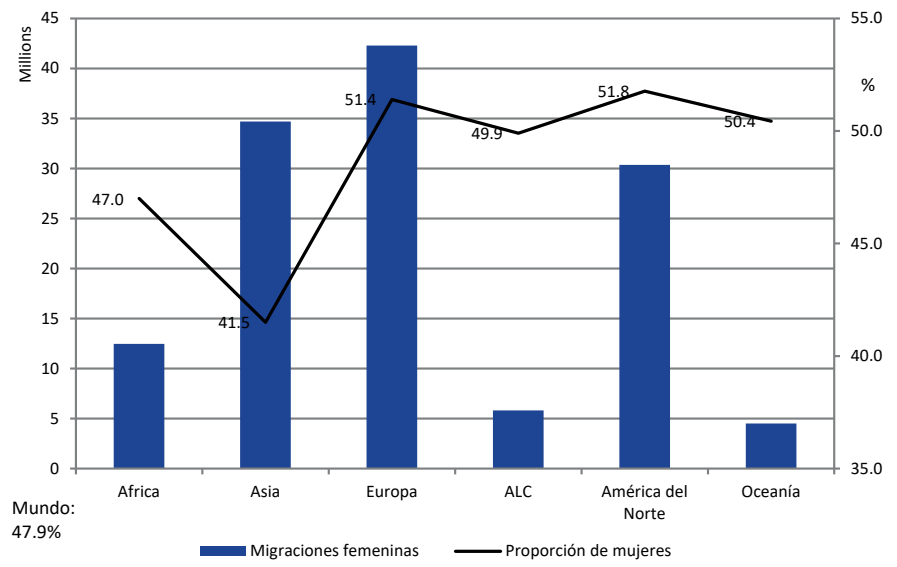

Fuente: United Nations, Deparment of Economic and Social Affairs, Populations Division (2019). ALC: América Latina y el Caribe.

Entre 1960 y 2013, la proporción de mujeres migrantes aumenta de $6.9 \%$ en la región América Latina y el Caribe (Gráfico $\mathrm{N}^{\circ} 2$ ), con valores de $44.7 \%$ en 1960, 48.4\% en 1980 (ONU, 2003), 49.8\% en 1990, $50.1 \%$ en $2000,51.5 \%$ en 2010 y $51.6 \%$ en 2013 (ONU, 2013). Sin embargo, esta proporción se reduce a 49.9\% en 2019 (ONU, 2019). El período de auge de 2000 a 2013 fue una consecuencia de la importancia de los flujos migratorios femeninos hacia España y entre varios países fronterizos de la región (Perú-Chile, Bolivia-Argentina, Paraguay-Argentina, Bolivia-Brasil, Colombia-Venezuela). Hay que destacar la importancia de las migraciones femeninas para sustentar el mercado de los trabajos de cuidado (care) en los países con poblaciones envejecidas, como España, Argentina y Chile; y algunos flujos migratorios están sumamente feminizados, como en actividades de comercio transfronterizo entre Bolivia y Brasil (Guimarães \& Baeninger, 2014). 
Gráfico №2 América Latina y el Caribe (ALC). Porcentaje de mujeres en el stock de inmigrantes1960-2019

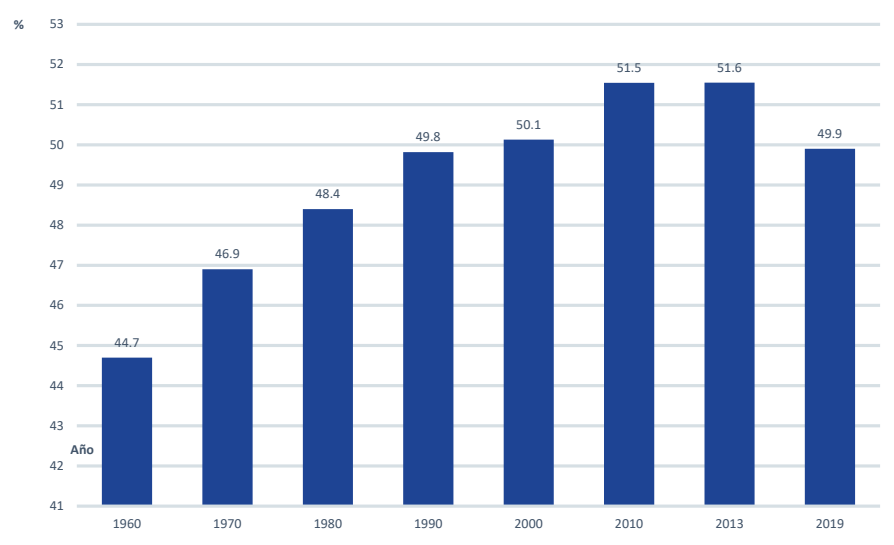

Fuente: United Nations, Deparment of Economic and Social Affairs, Populations Division (2003, 2011, 2013, 2019).

\section{- Los flujos migratorios: las tasas de migración neta}

Se pueden estimar los flujos migratorios netos de un país determinado, en un período dado, generalmente durante un año, al sustraer la población emigrante (salidas desde ese país) de la población inmigrante (entradas a ese país). Las tasas netas de migración se evalúan con los flujos netos en el numerador y con la población media del año en el denominador. Las tasas netas pueden ser positivas (predomina la inmigración) o negativas (predomina la emigración) y ofrecen una visión más dinámica de los flujos migratorios que los stocks, ya que éstos acumulan los inmigrantes sobre períodos mucho más largos e indefinidos.

En base a las tasas netas de migración de todos los países de América Latina entre 1950 y 2010, Victoria Prieto construye tipologías migratorias que muestran la heterogeneidad de la migración internacional de la región latinoamericana en generaciones recientes (Prieto, 2012). La mayoría de los flujos están compuestos por hombres y mujeres jóvenes, de menos de 30 años de edad, en todos los países y años. Pocos países registran emigración de mujeres, más bien de edades avanzadas (de Perú, El Salvador, Guatemala y Nicaragua). Todos los demás tipos de migración muestran un predominio masculino o bien un equilibrio de género (Prieto, 2012). Por lo tanto, los flujos netos de las migraciones femeninas expresan la gran heterogeneidad de la migración por edad y sexo en América Latina y no se puede sostener la idea de una feminización general de la migración (Prieto, 2012).

\subsection{Migraciones, familias y género}

Esta segunda parte se enfoca en varios ejemplos de migraciones femeninas y de sus determinantes. Se considera a las migraciones como una de las etapas de la entrada a la vida adulta, excluyendo la temática de las niñas y niños que migran con su familia. Tradicionalmente, en los estudios sobre la migración, se suponía que las mujeres solo acompañaban a los hombres migrantes. Sin embargo, las mujeres en edades adultas migran también de manera independiente por razones económicas (Lauby \& Stark 1987, Pfeiffer et al. 2007, Vause \& Toma 2015). El análisis se centra en algunas dimensiones de esas migraciones femeninas, como la presencia de mujeres latinoamericanas en el mercado matrimonial, la sexualidad de las migrantes, la maternidad y la vida familiar en migración, las relaciones de género y el empoderamiento de las mujeres migrantes, los mercados de trabajo transnacionales y de cuidados (care). Los ejemplos son muy diversos, y se observaron en España, en Francia y en países de América Latina, como Argentina, Bolivia, Brasil, Chile, Ecuador, Guatemala, México y Perú. A partir de estos casos, se presenta un esquema de las variables determinantes que relacionan las migraciones, las familias y el género. También se propone en la Figura $\mathrm{N}^{\circ} 1$ una herramienta sencilla y eficiente de recolección de datos y de análisis, la ficha AGEVEN (Cavagnoud, Baillet \& Cosío Zavala, 2019).

\section{- Mercados matrimoniales transnacionales}

Una consecuencia directa del aumento de las mujeres migrantes en los países de destino es que las pone en competencia, para la elección de un cónyuge, con las mujeres locales en los mercados matrimoniales. En algunos casos de emigración fuertemente masculina, los jóvenes migrantes son solteros cuando se van y regresan a su país de origen para unirse en su pueblo, como se observó en el norte de Guatemala con los migrantes a los Estados Unidos (Aragón, 2014). Sin embargo, en otros contextos, cuando un número significativo de mujeres inmigrantes se integra a la población de destino, los mercados matrimoniales se modifican. Así, se observa en España un fuerte aumento de los matrimonios entre españoles y extranjeras (tres veces más en 2010 que en 1998) y, en $70 \%$ de los casos, son hombres españoles emparejados con mujeres latinoamericanas (Domingo et al., 2014, p. 30). Existe una preferencia de los hombres españoles por las mujeres latinoamericanas (y también eslavas), por su "belleza" y "sensualidad", y porque son "amoro- 
sas" y "dóciles" (Roca Girona, 2014, p. 54). También "es importante señalar, según otras fuentes, los problemas de violencia familiar con la pareja y de separación como factores de motivación a emigrar" (Molano Mijangos, Robert \& García Domínguez, 2012, citado por Cavagnoud, 2014, p. 103). Además, los matrimonios entre nacionales y extranjeras permiten legalizar más fácilmente la situación migratoria de la pareja y de su descendencia.

\section{- Género y migración}

La necesidad de combinar la investigación sobre género y migración incluye temas como las dinámicas familiares, los mercados laborales y los roles de género en las familias migrantes (Parrado \& Flippen, 2005; Hondagneu-Sotelo, 2003; Morokvasic-MüIler et al., 2003). También hay que interesarse a la modificación de las relaciones de género durante el movimiento migratorio, en términos de relaciones de poder dentro de las familias y de carga emocional (Parrado \& Flippen, 2005). Las mujeres migrantes ganan o pierden autonomía y poder, dependiendo de esos tres niveles, tanto en el lugar de origen como en el de destino (Parrado \& Flippen, 2005). Sin embargo, el sistema de género antecede a la migración, en la organización de las relaciones entre los sexos construidas socialmente, y como un sistema jerárquico que otorga a los hombres un valor más alto que a las mujeres (Zavala de Cosío, 2010). A lo largo de las migraciones, las normas de feminidad y de masculinidad las acompañan, y se renuevan de acuerdo a las condiciones de salida, del traslado, de recepción, de integración y de discriminación en las sociedades de origen y destino.

En base a los resultados empíricos de los estudios de casos, se observan las reconfiguraciones de las vidas de hombres y mujeres a lo largo de las migraciones. Se destacan varios ejemplos de transformaciones familiares y de género según el sexo, como el control asimétrico de la sexualidad en la migración (Marroni, 2009), los nuevos roles y responsabilidades (Guimarães \& Baeninger, 2014), la interferencia de las dinámicas familiares con la dominación de género (Rosas, 2014), la masculinidad en el contexto indígena de los migrantes internacionales (Perraudin, 2014), la autonomía, el poder de decisión y el empoderamiento (Pagnotta, 2014), la maternidad a distancia (Cavagnoud, 2014), la contribución económica al hogar y el reconocimiento de los nuevos roles (Acosta \& Setién, 2014), así como los efectos de la crisis económica en los mercados laborales segmentados por el género (Pedone et al., 2018).

\section{- La sexualidad en la migración}

La sexualidad durante la migración es un tema importante de investigación, especialmente en contextos donde los hombres son mayoría y migran solos, sin sus familias. La asimetría del control de género es evidente para los migrantes mexicanos de Atlixco (Puebla) en los Estados Unidos: falta de control para los hombres migrantes y estricto control social de la sexualidad femenina durante la ausencia del cónyuge (Marroni, 2009, p. 213). La sexualidad fuera de la unión es aceptada para los hombres en los contextos migratorios, cuando las mujeres, si está el esposo ausente, se controlan estrictamente por parte de la comunidad, la familia y el entorno social en general (Perraudin, 2014).

\section{- Género, familia y empoderamiento}

Las migraciones se organizan a la par de las decisiones familiares, lo que origina cambios en las estructuras de los hogares y en los arreglos residenciales, según el género y las generaciones, y varios autores recomiendan tomar en cuenta las relaciones familiares para entender y explicar la migración (Pfeiffer et al., 2007). También se afirma que las obligaciones familiares de las mujeres siempre son un factor central, independientemente de la migración (Pessar, 2000). Algunas investigaciones analizan esas interacciones entre la migración y la familia, al abordar las relaciones entre los ciclos de vida familiar y las trayectorias de movilidad, por ejemplo de las mujeres bolivianas en Brasil (Guimarães \& Baeninger, 2014); la dominación de género, el empoderamiento (autonomía, dominación masculina, desigualdad, poder de decisión) y la función económica, con los ejemplos de familias peruanas en Argentina (Rosas, 2014), de familias otomís de la Ciudad de México (Perraudin, 2014) y de familias ecuatorianas en Francia (Pagnotta, 2014). Otros temas son la maternidad alejada y la redefinición de los roles de pareja y de parentesco a distancia, en cuanto al cuidado de las hijas y de los hijos por miembros de la familia (Cavagnoud, 2014). Estos ejemplos confirman que las relaciones entre las dinámicas familiares y de género son una dimensión fundamental de la movilidad, ya que la impulsan, la revierten o la modifican.

El estudio de las migraciones bolivianas en Brasil muestra que las trayectorias migratorias están fuertemente ligadas a las dinámicas familiares de las mujeres migrantes, e inversamente, sus ciclos de vida familiar están influidos por las etapas migratorias (Guimarães \& Baeninger, 2014). Por lo tanto, los cursos de vida familiares y migratorios son in- 
separables y deben estudiarse simultáneamente: "la planificación del ciclo de vida, tanto individual como familiar, es la cuestión central que define las trayectorias migratorias. Los usos de los espacios, conforme a esa planificación, orientan la movilidad de las mujeres migrantes" (Guimarães \& Baeninger, 2014, p. 209). En el contexto de la migración, las relaciones de género cambian inevitablemente, dadas las nuevas condiciones de vida y de organización familiar. De esa forma, el empoderamiento de las mujeres puede aumentar, pero no siempre es así, cuando los hombres se benefician de la migración para restablecer su rol de proveedor económico, lo que "satisface expectativas de ambos [cónyuges], y no sólo masculinas" (Rosas, 2014, p. 247). Igualmente, se observa cómo se fortalece la masculinidad de los hombres otomís que migran a los Estados Unidos por medio del envío de remesas (Perraudin, 2014). La desigualdad de género aumenta o se reduce, según los contextos sociales, políticos, económicos y culturales de la migración.

La emigración al extranjero (a Argentina, Brasil, España, Italia) de madres de la ciudad de El Alto (Bolivia) duplica la figura materna con: "la madre biológica que ha migrado para mejorar la movilidad social de la familia y la madre doméstica de la nueva vida cotidiana de los hijos, a cargo de la responsabilidad de sus estudios y de su educación" (Cavagnoud, 2014 , p. 109). Esas madres de la vida cotidiana son a menudo las abuelas. La emigrante transnacional que deja a sus hijas e hijos en el lugar de origen es considerada como una "mala madre" a pesar de las remesas para la familia (Cavagnoud, 2014). De manera asimétrica, el envío de remesas consolida la masculinidad de los varones por qué corresponde al rol tradicional de proveedor económico, pero para las mujeres no se valora igual, ya que no se considera como un rol central de la figura femenina y está lejos de compensar el abandono de los roles maternos. En consecuencia, las normas de género fijan en cada momento el valor de las responsabilidades de las mujeres migrantes y, a pesar de la adquisición de nuevos roles familiares y sociales, determinan la escasez de beneficios en cuanto a la igualdad de género, a la autonomía y al empoderamiento femeninos durante las migraciones.

\section{- Mercados de trabajo transnacionales}

La actividad económica de las mujeres migrantes depende de la segmentación de género en los mercados laborales. Por ejemplo, en Corumbà, Brasil, las comerciantes bolivianas se integran rápidamente a las actividades de ventas, un sector que les propor- ciona ingresos, y un estatus apreciado (Guimarães, Baeninger, 2014). Sin embargo, aunque emplea a muchas mujeres migrantes, el trabajo doméstico y de cuidado (care) en España y Chile es poco apreciado socialmente (Acosta \& Setién, 2014).

Por otra parte, en 2008 en España, la fuerte crisis económica provocó altas tasas de desempleo en la población inmigrante (Vidal-Coso \& Vono, 2014). Las negociaciones de género elaboraron estrategias familiares de retorno al país de origen, según el sexo, la edad, la escolaridad y la edad de las hijas e hijos (Pedone et al., 2014) y reconfiguraron la distribución de las tareas domésticas y de cuidado dentro de los hogares. A pesar de la crisis, las poblaciones latinoamericanas se quedaron mayoritariamente en España, o se trasladaron a otro país europeo. Aunque el flujo de retorno al país de origen aumentó, las redes migratorias están bien establecidas, muchas y muchos descendientes de migrantes tienen la nacionalidad del país de destino y allá han construido sus vidas.

\subsection{Las variables determinantes de las migracio- nes femeninas}

A partir de los resultados anteriores, se propone un esquema de análisis que comprende las variables determinantes de las migraciones en relación con las dinámicas familiares y de género. Se centran en cinco temáticas principales: la vida matrimonial, la sexualidad, la maternidad, los arreglos familiares y el trabajo, que se pueden analizar con una perspectiva de género antes, durante y después de las migraciones.

a) La vida matrimonial: Es indispensable seguir a lo largo de las biografías de las migrantes su trayectoria matrimonial. Las principales preguntas son: ¿Migraron como solteras o unidas? ¿Cuándo y dónde entraron en unión? ¿La unión se produce en el país de origen o de destino? ¿Quiénes son y dónde están los cónyuges? ¿Hay separaciones, divorcios, viudez? ¿Se vuelven a unir después de una ruptura de unión? Es indispensable estudiar la segmentación del mercado matrimonial transnacional (como en el ejemplo de las latinoamericanas en España que tienen la preferencia de los varones en el mercado matrimonial (Domingo et al. 2014; Roca Girona, 2014)). Pero cada situación particular debe ser estudiada, ya que el empoderamiento y agencia de las mujeres migrantes va a depender estrechamente del desarrollo de su trayectoria matrimonial a lo largo de la vida. 
b) La sexualidad durante la migración: es importante estudiar las nuevas normas sexuales durante las migraciones, porque van a reflejar las nuevas relaciones de género. En los ejemplos de hombres migrantes a los Estados Unidos, ellos se van y ellas se quedan (como las esposas de migrantes de Atlixco [Puebla, México] y de Otomís de la Ciudad de México). Se ha observado una fuerte asimetría durante la ausencia del cónyuge, sin control de la sexualidad de los hombres migrantes y con un fuerte control social de la sexualidad femenina por las familias de origen, de alianza y las comunidades (Marroni, 2009; Perraudin, 2014). Hay que examinar las consecuencias sobre las desigualdades de género, las discriminaciones y las situaciones de violencia doméstica y social.

c) La maternidad de las mujeres migrantes: en cada tipo de flujo migratorio, es necesario conocer la situación familiar y reproductiva de las mujeres migrantes en todo momento ¿Migran solteras o unidas? ¿Migran, solas o acompañadas? ¿Tienen hijas o hijos? ¿Se quieren embarazar para formar una familia? ¿Quién asume la crianza de sus hijas e hijos? Dependiendo de esas situaciones, del lugar de nacimiento y de la edad de sus hijas e hijos, la demanda de servicios materno-infantiles y educativos se tiene que determinar en el lugar de origen, durante la trayectoria migratoria y en el lugar de destino, lo que multiplica las presiones sobre las mujeres migrantes. Si ellas dejan a las hijas y a los hijos en el lugar de origen ¿Quiénes asumen los roles parentales? ¿Qué repercusiones existen en la autonomía y agencia de las mujeres migrantes? ¿Cuáles son las consecuencias, que pueden ser violentas, de las separaciones entre la pareja, los parientes, las y los hermanos?

d) Los arreglos familiares: las trayectorias migratorias son inseparables de los ciclos de vida familiar, etapa por etapa de la migración. Es así como "El ciclo de vida, especialmente casamiento y nacimiento de los hijos, es planificado en función de las etapas migratorias transitadas y de los usos estratégicos de cada uno de los espacios" (Guimarães y Baeninger, 2014, p. 210). La cuestión, en cada momento es observar las relaciones de parentesco de los que viven en diferentes hogares y lugares, y sus intercambios económicos y familiares. El empoderamiento femenino aumenta o se reduce en los diferentes momentos de las trayectorias migratorias y familiares (Cavagnoud, 2014; Guimarães \& Baeninger, 2014; Rosas, 2014; Perraudin, 2014; Pagnotta, 2014). d) Los mercados de trabajo transnacionales: las nuevas ocupaciones económicas de las mujeres migrantes necesitan sacrificios personales, para asegurar el bienestar de las hijas e hijos a la distancia (como en el caso de las bolivianas en España). Durante las trayectorias migratorias de las mujeres, las redes de apoyo familiares y sociales se destruyen y se recomponen, lo que implica grandes vulnerabilidades frente a los recursos y servicios a los que tienen derecho. Los períodos migratorios están relacionados con las etapas sucesivas de la vida laboral, en relación con los cursos de vida familiares y reproductivos. Surgen nuevos roles y obligaciones dentro de las familias en función de los mercados de trabajo transnacionales, tales como el mercado internacional de cuidados (care). También se distribuyen de manera diferente las tareas domésticas y de cuidado del hogar. Es indispensable analizar al mismo tiempo todas esas interrelaciones, con métodos biográficos, que son los más adecuados para poder hacerlo a lo largo del tiempo.

El esquema propuesto de variables determinantes de las familias migrantes se debe combinar con una perspectiva de género (Zavala de Cosío, 2010). Durante las migraciones, se considera el nivel de autonomía, de poder y de desigualdad entre hombres y mujeres, sobre el proceso de entrada en uniones y de disolución (separaciones, divorcios, viudez) y la toma de decisiones durante la vida matrimonial. ¿Quién y cómo se toman esas decisiones? Además, las normas se modifican según los contextos de las sociedades de origen y de destino, como las normas sexuales de las parejas separadas por la migración, que revelan el grado de autonomía, empoderamiento, libertades y derechos de los hombres y de las mujeres, que sean migrantes o no. Los arreglos familiares y la maternidad se modifican en cada una de las etapas migratorias, así como los recursos económicos de la familia: ¿quiénes son los y las responsables de los ingresos económicos de la familia? ¿el jefe de hogar? ¿la esposa del jefe? ¿las jefas de hogares monoparentales? ¿otros parientes? ¿quién se encarga del cuidado de las hijas e hijos dependientes? Por otra parte, está el control de los ingresos: ¿cómo se usan? ¿quién decide? ¿cuál es el destino de las remesas? ¿quién las usa y para qué?

La autonomía y el poder de decisión de las mujeres son centrales en las respuestas a esas preguntas. Además, hay que analizar el tipo de relaciones conyugales y el clima familiar: ¿cuál es el grado de dominación y desigualdad según el sexo? las relaciones ¿son complementarias? o ¿más bien iguali- 
tarias? ¿hay o no hay violencia doméstica? En resumen, la perspectiva de género en el campo de las dinámicas familiares y de las migraciones aporta nuevas preguntas de investigación, sobre el grado de autonomía, de empoderamiento, de las libertades y derechos de las mujeres con respecto a los varones, y en relación con las generaciones de madres, padres, hijas, hijos y otros parientes. Entre los diferentes modelos de familia, desde las que tienen relaciones muy desiguales y patriarcales hasta las que registran relaciones más igualitarias, hemos visto que las migraciones femeninas no son suficientes para pasar de un modelo al otro, a pesar de todas las transformaciones que implican para las mujeres migrantes, en términos de vida familiar y de roles económicos y sociales.

\subsection{Métodos biográficos}

Para analizar a profundidad las migraciones femeninas, una propuesta metodológica se basa en las historias de vida, con la observación de todos los cambios residenciales y de las trayectorias familiares, matrimoniales, reproductivas y laborales, vinculadas al poder de decisión, la autonomía, los roles y los derechos según el género. Cada vez que cambia la situación familiar, se observan los cambios residenciales; con cada movilidad residencial, se analizan las transformaciones familiares, laborales y de género (Cavagnoud, Baillet \& Cosío Zavala, 2019). Se pueden combinar los datos cuantitativos de encuestas realizadas en los lugares de migración con entrevistas retrospectivas, como en el trabajo de Roberta Guimarães y Rosana Baeninger (2014) en la frontera de Bolivia y Brasil, y en la encuesta de Carolina Rosas (2014) sobre la migración peruana a Buenos Aires (Rosas, 2014). En la parte cualitativa de las metodologías mixtas (Roberta Guimarães \& Rosana Baeninger, Carolina Rosas) así como en las investigaciones etnográficas de Anna Perraudin (2014), Chiara Pagnotta (2014) y Robin Cavagnoud (2014) se realizaron historias de vida, entrevistas y observación participante.

Además de los ejemplos anteriores, se puede señalar unas encuestas biográficas, las Encuestas Demográficas Retrospectivas (EDER) de 1998, 2011 y 2017 realizadas en México (Coubs, Zavala \& Zenteno, 2005; Coubès, Solís \& Zavala, 2016). Con datos de la EDER de 2011, Sebille analizó detalladamente las etapas de la historia familiar y matrimonial de mujeres y varones migrantes (Sebille, 2016). Otros ejemplos de investigaciones han utilizado las fichas AGEVEN para relacionar las historias de vida de la EDER con entrevistas a profundidad (Cavagnoud,
Baillet \& Cosío Zavala, 2019). Los resultados de las investigaciones mencionadas aquí arriba permiten observar cómo los períodos migratorios se entrecruzan con las etapas sucesivas de la vida familiar, escolar y laboral. Cómo surgen nuevos roles y obligaciones dentro de las familias, y cómo el movimiento migratorio modifica las relaciones de género y de generaciones. Por lo tanto, los métodos biográficos son muy adecuados para una observación a lo largo del tiempo de las trayectorias migratorias, familiares, laborales y de género, con cuestionarios cuantitativos, historias de vida cualitativas o ambos métodos combinados.

En la Figura $\mathrm{N}^{\circ} 1$ se propone un modelo de ficha AGEVEN para las encuestas sobre familia, migraciones y género, en base al modelo ya mencionado, con fichas sencillas de realizar y que muestran mucha información. Hay una gran cantidad de ejemplos de su aplicación con buenos resultados (Cavagnoud, Baillet \& Cosío Zavala, 2019). Se propone hacer entrevistas a las mujeres, pero también a los varones. Se colocan en la ficha retrospectivamente todos los cambios de residencia, los eventos familiares, laborales y migratorios, cada vez que suceden, edad por edad, año por año, desde el año de nacimiento hasta la edad en el momento de la encuesta. Se empieza por todos los cambios residenciales. Se describe el contexto en cada período de la vida, a nivel macro y meso, y las situaciones importantes económicas y sociales, el contexto migratorio, las redes de apoyo y las discriminaciones. Cuando se producen eventos y cambios, se puntualizan las relaciones de género (empoderamiento, autonomía, libertades y derechos) a nivel de las familias y de los individuos. También se listan los miembros cohabitantes del hogar que sean familiares o no, y los que no cohabitan, el clima familiar con la calidad de las relaciones y las situaciones de violencia. Y para la historia de vida de Ego (la persona entrevistada), se mencionan los eventos de la vida escolar, de la vida laboral en el mercado de trabajo y también del trabajo doméstico no remunerado. Se listan todos los eventos matrimoniales (uniones, sexualidad), la fecundidad y la vida reproductiva. Con este cuadro completo, es posible distinguir los eventos en cada momento, los puntos de inflexión cuando hay eventos trascendentales, sus relaciones con las situaciones de género, y se pueden explicar las interrelaciones entre familia, migraciones y género. La ficha AGEVEN es a la vez una herramienta de recolección de las historias de vida y de análisis de los resultados de todas las observaciones (Cavagnoud, Baillet \& Cosío Zavala, 2019). 
Figura N¹ Ficha AGEVEN para eventos familiares, migratorios y género

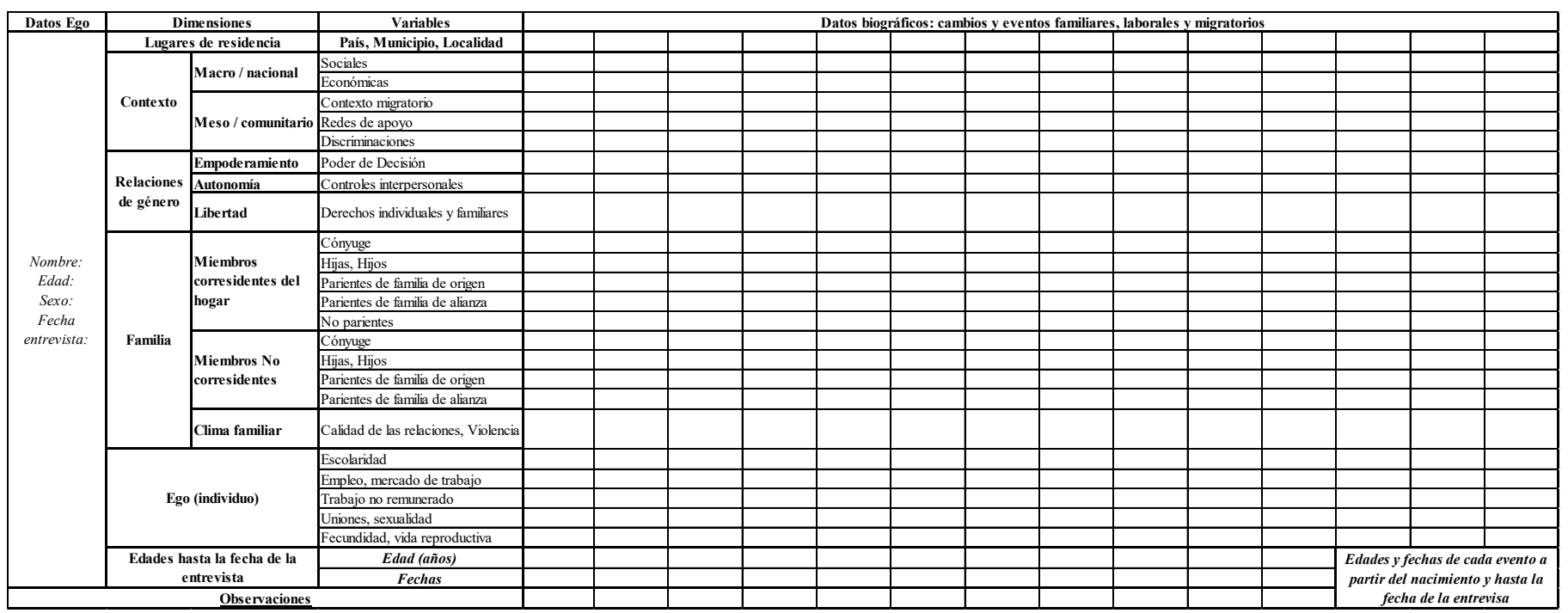

Fuente: United Nations, Deparment of Economic and Social Affairs, Populations Division (2003, 2011, 2013, 2019).

\section{Conclusiones}

Como reflexiones finales, aunque hemos observado que no se ha producido una feminización masiva de las migraciones latinoamericanas entre 2003 y 2019 , el análisis de las transformaciones familiares y de género completa significativamente las investigaciones sobre los movimientos migratorios. Los estudios de caso latinoamericanos revisados en este artículo han mostrado las consecuencias de la migración femenina sobre las relaciones de género y el empoderamiento femenino, en los mercados matrimoniales, la sexualidad de las migrantes, la vida reproductiva, los arreglos familiares y los mercados laborales transnacionales. Se han averiguado los cambios en la autonomía e independencia femenina, en los roles y responsabilidades familiares, en la maternidad transnacional, en la participación económica de las mujeres, tanto en los países de destino como en los países de origen. Estos conceptos conducen a elaborar un esquema de variables determinantes de las relaciones entre familia, género y migraciones, y a elaborar una herramienta, con una perspectiva biográfica, para seguir las etapas de las historias de vida a lo largo del tiempo. La ficha AGEVEN es un método biográfico para el trabajo de campo, que se adapta a la temática que interesa en este trabajo (Figura $N^{\circ} 1$ ), y es una herramienta sencilla y eficiente, ampliamente utilizada (Cavagnoud, Baillet \& Cosío Zavala, 2019).

Entre los resultados importantes de este artículo se muestra que, a pesar del aumento en la participación económica de las mujeres migrantes, su situación fa- miliar no se ve mejorada en términos de igualdad de género, de poder de decisión, ni de reconocimiento de las nuevas atribuciones adquiridas con grandes sacrificios personales, especialmente para asegurar el bienestar de sus hijas e hijos. La desigualdad de género en las sociedades latinoamericanas de origen sigue ejerciéndose a través de las fronteras, al tiempo que se remodela durante el proceso de integración de las mujeres migrantes al país de destino, con nuevos roles y obligaciones.

Habría que añadir a estos trabajos, realizados en la primera década del Siglo XXI, las evoluciones más recientes de las migraciones latinoamericanas. Por ejemplo, desde 2008, se ha reducido significativamente la migración de México hacia los Estados Unidos, a partir de la crisis económica mundial, aunada al control militar en la frontera norte de México. Hay que investigar cuáles son las consecuencias de ese cambio sobre las relaciones de género en las familias migrantes. Otro ejemplo sería el de las familias de los "retornados", migrantes devueltos por las autoridades norteamericanas a México. Algo ya se sabe sobre sus condiciones de vida en tierras mexicanas (Rivera Sánchez, 2019), pero falta investigar a profundidad sus historias familiares y migratorias con perspectiva de género. Otro tema candente es el aumento de las migraciones de familias centroamericanas hacia los Estados Unidos. Por las nuevas políticas de represión a las migraciones y al asilo, por parte de los gobiernos estadunidenses y mexicanos, muchas familias se tienen que quedar en las regiones fronterizas del norte y del sur de México, en campos de retención desprovistos de los servicios 
básicos, en condiciones de hacinamiento y de violaciones de los derechos humanos. ¿Cómo se consideran a las familias en esos centros? Sabemos que impera la violencia en esos lugares, sobre todo la violencia en contra de las mujeres, y notablemente la violencia sexual. Pero ¿qué hacen las autoridades y las asociaciones de ayuda para apoyar a esas familias migrantes? ¿Qué políticas públicas y privadas se tienen que llevar a cabo para proteger a las mujeres, a sus hijos y a sus familias?

Un cambio imprevisto y fundamental es el de las emigraciones desde Venezuela. En 2017/2018, cerca de 3 millones de venezolanos vivían fuera de su país, un crecimiento impresionante, comparado a los 700 mil emigrantes venezolanos en 2015 (Gandini, Lozano \& Prieto, 2019, p. 45). Según los datos recientes, se estima que, entre ellos, la relación de masculinidad asciende a casi 103 hombres por cada 100 mujeres en 2017, y a 109 hombres por cada 100 mujeres en 2018, los cuales salieron principalmente para buscar trabajo. Eso contrasta con la situación de 2006, cuando las migraciones desde Venezuela eran principalmente femeninas (Gandini et al., 2019, p. 49). Este nuevo movimiento abarca preguntas de investigación, desconocidas y urgentes de esclarecer.

Finalmente, un tema pendiente y central es el de la adecuación de las políticas públicas para dirigirse específicamente a las familias migrantes, a las parejas, a las niñas y a los niños. Por ejemplo, la separación de los menores migrantes con sus padres y madres, en los Estados Unidos y en las fronteras norte y sur de México, es una grave violación a los derechos humanos y personales. Además, en los tiempos de crisis, los y las migrantes necesitan más que nunca una protección familiar, especialmente en dirección de las mujeres migrantes. $Y$ a todo eso se añade ahora la pandemia del COVID-19, otro factor más de discriminación y precariedad en contra de las poblaciones migrantes.

Sin embargo, en todas las regiones del mundo, y también en América Latina, las mujeres, los hombres y las familias migran intensamente, cada vez más lejos, y siempre con la esperanza de una vida mejor, aun cuando viven duras experiencias.

\section{Referencias bibliográficas}

- Acosta González, Elaine, \& Setien Santamaría, María Luisa. (2014). Entre las "chicas para todo" y las "nanas de toda la vida". Representaciones y valoración social de las cuidadoras domésticas de origen inmigrante en España y Chile. In Za- vala de Cosío, María Eugenia \& Rozée, Virginie (Coords.), El género en movimiento, familias y migraciones (pp. 193-220). Ciudad de México, México: El Colegio de México.

- Aragón, Argan. (2014). Migrations clandestines d'Amérique centrale vers les Etats-Unis. Préface de Catherine Withol de Wenden. Prix de thèse des PSN en 2014. Paris, France: Presses Sorbonne nouvelle (PSN).

- Cavagnoud, Robin. (2014). El impacto de las migraciones internacionales de mujeres bolivianas en el trayecto de vida de sus hijos no migrantes: el caso de El Alto. In Zavala de Cosío, María Eugenia \& Rozée, Virginie (Coords.), El género en movimiento, familias y migraciones (pp. 139158). Ciudad de México, México: El Colegio de México.

- Cavagnoud, Robin, Baillet, Julie, \& Cosío Zavala, María Eugenia (2019). L'usage de l'approche qualitative dans les études de populations : apports et enjeux méthodologiques, Cahiers Québécois de Démographie, 48, 1, pp. 27-51. Montréal, Canadá : Association des démographes du Québec. https://doi.org/10.7202/1073339ar.

- Coubès, Marie-Laure, Zavala de Cosío, María Eugenia, \& Zenteno, René. (2005), Cambios demográficos y sociales en México del Siglo XX: una perspectiva de historias de vida, México: Miguel Angel Porrúa.

- Coubès, Marie-Laure, Solís, Patricio \& Zavala de Cosío, María Eugenia (Coords.). (2016). Generaciones, cursos de vida y desigualdad social. Ciudad de México y Tijuana: El Colegio de México y El Colegio de la Frontera Norte.

- Donato, Katharine, \& Gabaccia, Donna. (2015). Gender and International Migration: From Slavery Era to the Global Age. Recuperado de: https://www.russellsage.org/publications/gender-and-international-migration

- Domingo, Andreu, Esteve, Albert, \& Bueno, Xania. (2014). El rapto de las Latinas: Migración latinoamericana y mercados matrimoniales en España. In Zavala de Cosío, María Eugenia \& Rozée, Virginie (Coords.), El género en movimiento, familias y migraciones (pp. 41-66). Ciudad de México, México: El Colegio de México.

- Gandini, Luciana, Lozano-Ascencio, Fernando, \& Prieto, Victoria. (2019). Crisis y Migración de 
la población venezolana. Entre la desprotección y la seguridad jurídica en Latinoamérica, México: UNAM, Universidad Nacional Autónoma de México. Recuperado de: https://www.sdi.unam.mx/ docs/libros/SUDIMER-CyMdPV.pdf

- Guimarães, Roberta, \& Baeninger, Rosana. (2014). Espacios migratorios en la Frontera: inmigración boliviana y género. In Zavala de Cosío, María Eugenia \& Rozée, Virginie (Coords.), El género en movimiento, familias y migraciones (pp. 261-294). Ciudad de México, México: El Colegio de México.

- Hondagneu-Sotelo, Pierrette, (2003). Gender and U.S. immigration: Contemporary trends. Berkeley, USA: University of California.

- Lauby, Jennifer, \& Stark, Oded. (1987). Individual migration as a family strategy: young women in the Philippine. Cambridge, Mass. USA: Center for Population Studies, Migration and Development Program, Harvard University.

- Marroni, María da Gloria. (2009). Frontera perversa, familias fracturadas. Los indocumentados mexicanos y el sueño americano. Puebla, México: Benemérita Universidad Autónoma de Puebla, Instituto de Ciencias Sociales y Humanidades. http://www.gimtrap.org/files/375.pdf

- Molano Mijangos, Adriana, Robert, Elisabeth, \& García Domínguez, María de los Ángeles. (2012). Cadenas globales de cuidados: síntesis de resultados de nueve estudios en América Latina y España, Santo Domingo: ONU Mujeres. Recuperado de: http://www.unwomen.org/ wp-content/uploads/2012/09/sintesis_de_nueve_estudios.pdf

- Morokvasic- Müller, Mirjana, Erel, Umut, \& Shinozaki, Kyoko (Eds.). (2003). Crossing Borders and Shifting Boundaries. Vol. 1: Gender on the Move. Opladen, Alemania: Leske \& Budrich.

- ONU. (2003). United Nations, Department of Economic and Social Affairs. Population Division. International Migration Report 2002. ST/ESA/ SER.A/220. New York, USA: United Nations.

- ONU. (2011). United Nations, Department of Economic and Social Affairs, Population Division. Trends in International Migrant Stock: Migrants by Age and Sex (United Nations database, POP/DB/MIG/Stock/Rev.2010). Recuperado de: http://www.un.org/en/development/desa/popula- tion/migration/data/index.shtml

- ONU. (2013). United Nations, Department of Economic and Social Affairs, Population Division. Trends in International Migrant Stock: Migrants by Age and Sex (United Nations database, POP/DB/MIG/Stock/Rev.2013). Recuperado de: http://www.un.org/en/development/desa/population/migration/data/estimates2/estimatesage. shtml

- ONU (2019). United Nations, Department of Economic and Social Affairs, Population Division. International Migrant Stock: Migrants by Age and Sex (United Nations database, POP/DB/MIG/ Stock/Rev.2019). Recuperado de: https://www. un.org/en/development/desa/population/migration/data/estimates2/estimates19.asp

- Pagnotta, Chiara. (2014). ¡Con mi papi no se puede contar! Relatos sobre las relaciones de género de los migrantes ecuatorianos en París. In Zavala de Cosío, María Eugenia \& Rozée, Virginie (Coords.), El género en movimiento, familias y migraciones (pp. 359-373). Ciudad de México, México: El Colegio de México.

- Parrado, Emilio \& Flippen, Chenoa. (2005). Migration and Gender among Mexican Women, American Sociological Review, Vol. 70, August 2005, pp. 606-632

- Pedone, Claudia, Echeverri, María Margarita \& Gil, Sandra. (2014). Entre dos orillas. Cambios en las formas de organización de las familias migrantes latinoamericanas en España en tiempos de crisis global. In Zavala de Cosío, María Eugenia \& Rozée, Virginie (Coords.), El género en movimiento, familias y migraciones (pp. 109138). Ciudad de México, México: El Colegio de México.

- Perraudin, Anna. (2014). Migrar para afianzar las masculinidades. La renegociación de las relaciones de género de la Ciudad de México a Estados Unidos: el caso de una población indígena. In Zavala de Cosío, María Eugenia \& Rozée, Virginie (Coords.), El género en movimiento, familias y migraciones (pp. 333-357). Ciudad de México, México: El Colegio de México.

- Pessar, Patricia R. (2000). The linkage between the household and workplace of Dominican women in the U.S. International Migration Review, XVIII (4), 2000. pp. 1188-1211. 
- Pfeiffer, Lisa, Richter, Susan, Fletcher, Peri \& Taylor J. Edward. (2007). Gender in Economic Research on International Migration and Its Impacts: A Critical Review. Recuperado de: http:// essays.ssrc.org/remittances_anthology/wp-content/uploads/2009/08/Topic_7_Pfeiffer.pdf

- Prieto, Victoria. (2012). El componente demográfico de las migraciones exteriores de América Latina, 1950-2050. Tesis doctoral. Barcelona, España: Universidad Autónoma de Barcelona.

- Rivera Sánchez, Liliana. (2019). Volver a casa. migrantes de retorno en América Latina. Debates tendencias y experiencias divergentes. Ciudad de México, México: El Colegio de México.

- Roca Girona, Jordi. (2014). Buscar el amor, redefinir las relaciones de género. In Zavala de Cosío, María Eugenia \& Rozée, Virginie (Coords.), El género en movimiento, familias y migraciones (pp. 67-83). Ciudad de México, México: El Colegio de México.

- Rosas, Carolina. (2014). ¿Cómo se toman las decisiones en el hogar? Cambios y continuidades en las voces de mujeres y hombres peruanos en Buenos Aires. In Zavala de Cosío, María Eugenia \& Rozée, Virginie (Coords.), El género en movimiento, familias y migraciones (pp. 295331). Ciudad de México, México: El Colegio de México.

- Sebille, Pascal. (2016). La migración en México ¿Una historia de familia? ¿Un asunto de género? En Coubès, Marie-Laure, Solís, Patricio \& Zavala de Cosío, María Eugenia (Coords.). (2016), Generaciones, cursos de vida y desigualdad social (pp. 255-279). Ciudad de México y Tijuana: El Colegio de México y El Colegio de la Frontera Norte.

- Schrover, Marlou. (2013). Feminization and problematization of migration: Europe in the nineteenth and twentieth centuries'. In Hoerder, Dirk \& Kaur, Amarjit (Eds.), Proletarian and gendered mass migrations. A global perspective on continuities and discontinuities from the 19th to the 21st Centuries (pp. 103-131). Studies in Global Social History, 12/1. Leiden, Holanda: University of Leiden, Brill collection.

- Vause, Sophie, \& Toma, Sorana. (2015). Peut-on parler de féminisation des flux migratoires du Sénégal et de la République démocratique du Con- go? Population-F, 70 (1), 2015, pp. 41-68.

- Vidal-Coso, Elena \& Vono Vilhena, Daniela (2014). Equilibrios ocupacionales en los hogares de latinoamericanas en España durante las etapas de expansión y crisis económica, 1999-2011. In Zavala de Cosío, María Eugenia \& Rozée, Virginie (Coords.), El género en movimiento, familias y migraciones (pp. 161-192). Ciudad de México, México: El Colegio de México.

- Zavala de Cosío, María Eugenia. (2010). Familia y relaciones de género. In Ludec, Nathalie (Coord.). Identidades de Género en transformación en América latina: aportes europeos y americanos (XIX-XX) (pp. 145-166). Quito, Ecuador: Ediciones Abya-Yala.

- Zavala de Cosío, Maria Eugenia \& Rozée, Virginie (Coord.). (2014). El género en movimiento, familias y migraciones. Ciudad de México, México: El Colegio de México. 